\title{
Characteristics and pregnancy outcomes across gestational diabetes mellitus subtypes based on insulin resistance
}

\author{
Katrien Benhalima ${ }^{1} \cdot$ Paul Van Crombrugge ${ }^{2} \cdot$ Carolien Moyson $^{1} \cdot$ Johan Verhaeghe $^{3} \cdot$ Sofie Vandeginste ${ }^{4}$. \\ Hilde Verlaenen ${ }^{4}$. Chris Vercammen ${ }^{5}$. Toon Maes ${ }^{5}$. Els Dufraimont ${ }^{6}$. Christophe De Block ${ }^{7}$. Yves Jacquemyn ${ }^{8}$. \\ Farah Mekahli ${ }^{9} \cdot$ Katrien De Clippel $^{10}$ - Annick Van Den Bruel ${ }^{11}$. Anne Loccufier ${ }^{12}$. Annouschka Laenen ${ }^{13}$. \\ Caro Minschart ${ }^{1} \cdot$ Roland Devlieger $^{3} \cdot$ Chantal Mathieu $^{1}$
}

Received: 5 March 2019 / Accepted: 17 June 2019 / Published online: 23 July 2019

(C) Springer-Verlag GmbH Germany, part of Springer Nature 2019

\begin{abstract}
Aims/hypothesis This study aimed to determine the characteristics and pregnancy outcomes across different subtypes of gestational diabetes mellitus (GDM) based on insulin resistance.

Methods GDM subtypes were defined in 1813 pregnant women from a multicentre prospective cohort study, stratified according to insulin resistance, based on Matsuda index below the 50th percentile of women with normal glucose tolerance (NGT), during a 75 g OGTT at 24-28 weeks' gestation. GDM was diagnosed in $12.4 \%(n=228)$ of all participants based on the 2013 WHO criteria.

Results Compared with women with NGT (1113 [61.4\%] of the total cohort) and insulin-sensitive women with GDM (39 [17.1\%] women with GDM), women with GDM and high insulin resistance (189 [82.9\%] women with GDM) had a significantly higher BMI, systolic BP, fasting plasma glucose (FPG), fasting total cholesterol, LDL-cholesterol and triacylglycerol levels in early pregnancy. Compared with women with NGT, insulin-sensitive women with GDM had a significantly lower BMI but similar BP, FPG and fasting lipid levels in early pregnancy. Compared with women with NGT, women with GDM and high insulin resistance had higher rates of preterm delivery $(8.5 \%$ vs $4.7 \%, p=0.030)$, labour induction $(42.7 \%$ vs $28.1 \%, p<0.001)$, Caesarean section (total Caesarean sections: $28.7 \%$ vs $19.4 \%, p=0.004$; emergency Caesarean sections: $16.0 \%$ vs $9.7 \%, p=$ $0.010)$, neonatal hypoglycaemia $(15.4 \%$ vs $3.5 \%, p<0.001)$ and neonatal intensive care unit admissions $(16.0 \%$ vs $8.9 \%, p=$ 0.003). In multivariable logistic regression analyses using different models to adjust for demographics, BMI, FPG, HbA $1 \mathrm{c}$, lipid levels and gestational weight gain in early pregnancy, preterm delivery (OR 2.41 [95\% CI 1.08, 5.38]) and neonatal
\end{abstract}

Electronic supplementary material The online version of this article (https://doi.org/10.1007/s00125-019-4961-7) contains peer-reviewed but unedited supplementary material, which is available to authorised users.

Katrien Benhalima

katrien.benhalima@uzleuven.be

1 Department of Endocrinology, UZ Gasthuisberg, KU Leuven, Herestraat 49, 3000 Leuven, Belgium

2 Department of Endocrinology, OLV Ziekenhuis Aalst-Asse-Ninove, Aalst, Belgium

3 Department of Obstetrics \& Gynecology, University Hospital Gasthuisberg, KU Leuven, Leuven, Belgium

4 Department of Obstetrics \& Gynecology, OLV Ziekenhuis Aalst-Asse-Ninove, Aalst, Belgium

5 Department of Endocrinology, Imelda Ziekenhuis, Bonheiden, Belgium

6 Department of Obstetrics \& Gynecology, Imelda Ziekenhuis, Bonheiden, Belgium
7 Department of Endocrinology-Diabetology-Metabolism, Antwerp University Hospital, Edegem, Belgium

8 Department of Obstetrics \& Gynecology, Antwerp University Hospital, Edegem, Belgium

9 Department of Endocrinology, Kliniek St-Jan Brussel, Brussels, Belgium

10 Department of Obstetrics \& Gynecology, Kliniek St-Jan Brussel, Brussels, Belgium

11 Department of Endocrinology, AZ St-Jan Brugge, Brugge, Belgium

12 Department of Obstetrics \& Gynecology, AZ St-Jan Brugge, Brugge, Belgium

13 Center of Biostatics and Statistical Bioinformatics, KU Leuven, Leuven, Belgium 


\section{Research in context}

\section{What is already known about this subject?}

- Women with gestational diabetes mellitus (GDM) often have subclinical metabolic dysfunction prior to conception

- Heterogeneity of the physiological processes underlying hyperglycaemia might also exist among women with GDM

What is the key question?

- Are different physiological GDM subtypes based on different degrees of insulin resistance associated with different phenotypic characteristics, adverse pregnancy outcomes and glucose intolerance postpartum?

What are the new findings?

- GDM with high insulin resistance represents a more adverse metabolic profile, with a greater risk of adverse pregnancy outcomes

- Insulin-sensitive women with GDM had a similar phenotype to, and similar pregnancy outcomes as, women with normal glucose tolerance

- The need for insulin treatment during pregnancy and rate of glucose intolerance in the early postpartum period were not significantly different between the GDM subtypes

How might this impact on clinical practice in the foreseeable future?

- A more accurate stratification of GDM based on underlying physiological processes might help to better tailor management during pregnancy and long-term follow-up after delivery to prevent complications

hypoglycaemia (OR 4.86 [95\% CI 2.04, 11.53]) remained significantly higher in women with GDM and high insulin resistance compared with women with NGT. Insulin-sensitive women with GDM had similar pregnancy outcomes as women with NGT. The need for insulin treatment during pregnancy and the rate of glucose intolerance in the early postpartum period were not significantly different among the GDM subtypes.

Conclusions/interpretation GDM with high insulin resistance represents a more adverse metabolic profile with a greater risk of adverse pregnancy outcomes.

Keywords Gestational diabetes mellitus · Pregnancy outcomes · Subtypes, insulin resistance, 2013 WHO criteria

\author{
Abbreviations \\ BEDIP-N Belgian Diabetes in Pregnancy Study \\ FPG Fasting plasma glucose \\ GCT $\quad 50 \mathrm{~g}$ Glucose challenge test \\ GDM Gestational diabetes mellitus \\ ISSI-2 Insulin secretion-sensitivity index-2 \\ LGA Large for gestational age \\ NGT Normal glucose tolerance \\ NICU Neonatal intensive care unit
}

\section{Introduction}

Gestational diabetes mellitus (GDM) is an important modifiable risk factor for adverse pregnancy outcomes and is associated with an increased risk of developing type 2 diabetes in later life [1-3]. Women who develop GDM often already have a subclinical metabolic dysfunction prior to conception compared with women with normal glucose tolerance (NGT) [4]. Because of the significant decrease in insulin sensitivity in normal pregnancy, this predisposing baseline insulin resistance is further exacerbated and, in combination with beta cell dysfunction, results in the development of GDM [4].

More data are needed on whether GDM subtypes with different degrees of insulin resistance are associated with different phenotypic characteristics, and have a differential risk of adverse pregnancy outcomes and glucose intolerance postpartum. A North American study in 809 predominantly white women has suggested that heterogeneity in the physiological processes underlying hyperglycaemia exist among women with GDM, and that GDM with impaired insulin sensitivity might represent a greater risk of adverse pregnancy outcomes [5]. In that study, women with GDM were considered to have an insulin secretion or insulin sensitivity defect if either was below the 25th percentile of the distribution in women with NGT [5]. The physiological stratification of GDM subtypes 
now needs to be evaluated in other populations with larger sample sizes. In addition, a more extensive evaluation of clinical and biochemical variables in early pregnancy is needed to evaluate differences in phenotypic characteristics across different GDM subtypes. More data are also needed on the differential risk of adverse pregnancy outcomes and the risk of glucose intolerance in the early postpartum period across different GDM subtypes. Our aim was therefore to evaluate the characteristics, pregnancy outcomes and risk of glucose intolerance across GDM groups based on different degrees of insulin resistance compared with women with NGT from a large prospective cohort study.

\section{Methods}

The Belgian Diabetes in Pregnancy Study (BEDIP-N) was a multicentre prospective cohort study that has previously been described in detail [6-8]. The study protocol was approved by the institutional review boards of all participating centres. Participants provided informed consent before inclusion in the study. Women were enrolled at between 6 and 14 weeks of pregnancy. Those without impaired glucose tolerance or diabetes in early pregnancy (defined by ADA criteria) received both a non-fasting $50 \mathrm{~g}$ glucose challenge test (GCT) and a $75 \mathrm{~g} 2 \mathrm{~h}$ OGTT between 24 and 28 weeks of pregnancy. During the study, participants and healthcare providers were blinded to the result of the GCT. Therefore, all participants received an OGTT irrespective of the GCT result. The diagnosis of GDM was based on the International Association of Diabetes and Pregnancy Study Groups criteria, now commonly referred to as the $2013 \mathrm{WHO}$ criteria for GDM [6, 7]. Of all the BEDIP-N participants, $1813(90.3 \%)$ received both a GCT and an OGTT between 24 and 28 weeks of pregnancy, with 228 women diagnosed with GDM (prevalence of 12.4\%). Overall, 106 participants (5.3\%) discontinued the study before 24 weeks of pregnancy, half because of a medical reason [8]. We have recently shown that the threshold of the GCT would need to be reduced to at least $7.2 \mathrm{mmol} / \mathrm{l}$ to achieve sensitivity of $\geq 70 \%$ for GDM based on the 2013 WHO criteria [7]. Based on a GCT result of $\geq 7.2 \mathrm{mmol} / \mathrm{l}$ and the $75 \mathrm{~g}$ OGTT, women without GDM could be stratified into the following two groups: NGT on the antepartum OGTT with an abnormal preceding GCT result (abnormal GCT NGT group); and NGT on the OGTT with a normal preceding GCT result (normal GCT NGT group).

The ADA-recommended glycaemic targets were used for the treatment of GDM [9]. If targets were not met within 2 weeks after the start of lifestyle measures then insulin was started. Women with GDM were invited for an extra visit at 616 weeks postpartum to receive a $75 \mathrm{~g}$ OGTT, and the ADA criteria were used to define diabetes and glucose intolerance (impaired fasting glucose and/or impaired glucose tolerance) $[6,9]$.

Baseline characteristics and the obstetric history were collected in early pregnancy [6]. An ethnic minority background was defined as having at least one parent from a non-white origin. In early pregnancy and at 24-28 weeks of pregnancy, anthropometric measurements were obtained and several selfadministered questionnaires were completed [6]. BP was measured twice at $5 \mathrm{~min}$ intervals using an automatic BP monitor [6]. A woman was defined as being overweight if her BMI was $\geq 25 \mathrm{~kg} / \mathrm{m}^{2}$ and as obese at BMI $\geq 30 \mathrm{~kg} / \mathrm{m}^{2}$.

At the first visit between 6 and 14 weeks of pregnancy, a fasting blood test was performed to measure fasting plasma glucose (FPG), insulin, lipid profile (total cholesterol, HDLand LDL-cholesterol, triacylglycerols) and $\mathrm{HbA}_{1 \mathrm{c}}$. HOMA-IR and HOMA-B were measured in early pregnancy, as previously described [10]. At the time of the OGTT, the fasting lipid profile and $\mathrm{HbA}_{1 \mathrm{c}}$ were measured. Glucose and insulin were measured fasting and at 30, 60 and $120 \mathrm{~min}$. An increase in triacylglycerols was defined as the difference between fasting triacylglycerol levels in early pregnancy and at the time of the OGTT [6].

To define GDM subgroups based on different degrees of insulin resistance, we used insulin and glucose levels during the $75 \mathrm{~g}$ OGTT at 24-28 weeks of pregnancy to calculate the Matsuda index, a whole-body measure of insulin sensitivity [11]. For the main analyses, we classified GDM in two different groups based on the Matsuda index: a GDM insulinresistant group, defined as a Matsuda index <50th percentile (P) of the NGT group; and a GDM insulin-sensitive group, defined as a Matsuda index above P50 of the NGT group. In the electronic supplementary material (ESM), we provide additional analyses of four GDM subgroups stratified according to the Matsuda index of $<$ P25, P25-P50, P50-P75 and $>$ P75 compared with women with NGT (ESM Tables 1-3). In addition, HOMA-IR and indices of beta cell function (HOMA$\mathrm{B}$, the insulinogenic index divided by HOMA-IR, the insulin secretion-sensitivity index-2 [ISSI-2] and the Stumvoll index) were measured, as previously described [10, 12-14].

The following pregnancy outcome data were collected: gestational age, pre-eclampsia (de novo $\mathrm{BP} \geq 140 / 90 \mathrm{mmHg}$ for $>20$ weeks with proteinuria or signs of end-organ dysfunction), gestational hypertension (de novo $\mathrm{BP} \geq 140 / 90 \mathrm{mmHg}$ for $>20$ weeks), type of labour and type of delivery with the indications, birthweight, macrosomia ( $>4 \mathrm{~kg}$ ), birthweight $\geq 4.5 \mathrm{~kg}$, large for gestational age (LGA) infant (defined as birthweight >P90 according to standardised Flemish birth charts adjusted for the sex of the baby and parity [15]), small for gestational age infant (defined as birthweight $<\mathrm{P} 10$ according to standardised Flemish birth charts adjusted for the sex of the baby and parity [15]), preterm delivery $(<37$ completed weeks), 10 min Apgar score, shoulder dystocia, neonatal respiratory distress syndrome, neonatal jaundice, congenital 
anomalies and admission to the neonatal intensive care unit (NICU) [6]. A glycaemic value of $<2.2 \mathrm{mmol} / 1$, irrespective of the need for i.v. administration of glucose and admission to the NICU, was considered as a neonatal hypoglycaemia across all centres. Admission to the NICU was decided by the neonatologist in line with the normal routine of each centre. Excessive weight gain during pregnancy was defined according to the 2009 Institute of Medicine guidelines [16]. Early weight gain was calculated as the difference in weight between the first prenatal visit and the time of the OGTT, while total weight gain was calculated as the difference in weight between the first prenatal visit and delivery.

Analyses of FPG at 6-14 weeks and the glucose measurements of the OGTT were conducted locally at each centre. Analyses of GCTs and insulin, lipid and $\mathrm{HbA}_{1 \mathrm{c}}$ levels were conducted centrally at the laboratory of UZ Leuven, and these results were not communicated to participants or healthcare providers during the study. Plasma glucose was measured using an automated colorimetric-enzymatic method on a Hitachi/ Roche Modular P analyser (Basel, Switzerland). Insulin was measured using an immunometric electrochemiluminescence immunoassay analyser (Roche Modular E170, Roche, Basel, Switzerland). $\mathrm{HbA}_{1 \mathrm{c}}$ was measured using a Tosoh Automated Glycohemoglobin Analyzer HLC-723G8 (Tokyo, Japan). Lipid levels were measured using the immunoassay analyser Cobas 8000 (Roche). Coefficients of variance were $1 \%$ for glucose, $6 \%$ for insulin, about $2 \%$ for lipids and $2 \%$ for $\mathrm{HbA}_{1 \mathrm{c}}$ in the laboratory of UZ Leuven.

Statistical analysis Characteristics and pregnancy outcomes were compared between each GDM subtype and the normal GCT NGT group. The frequency of glucose intolerance postpartum was evaluated in women with GDM. Continuous variables are presented as means \pm SD if normally distributed and as medians (interquartile range) otherwise, and categorical variables as percentages. The $\chi^{2}$ test (or Fisher's exact test in case of small cell frequencies) was used for comparing categorical variables between groups. The Mann-Whitney $U$ test or Kruskal-Wallis test was used to compare continuous variables among two or multiple groups, respectively. Additional comparisons of pregnancy outcomes between the GDM insulin-resistant group (and the GDM high insulin resistance group in the additional analyses in ESM Table 3) and the NGT group were performed using multivariable logistic regression analyses adjusted for the following confounders: model 1 : unadjusted model; model 2: adjusted for demographics (centre, age, ethnicity, parity, education and smoking before pregnancy); model 3: model 2 plus adjusted for first-degree family history of diabetes, previous history of GDM and BP in early pregnancy; model 4: model 3 plus adjusted for maternal BMI and waist circumference in early pregnancy; model 5: model 4 plus adjusted for fasting lipids (total cholesterol, LDLcholesterol and triacylglycerols), FPG and $\mathrm{HbA}_{1 \mathrm{c}}$ in early pregnancy; model 6: model 5 plus adjusted for gestational weight gain until the OGTT at 24-28 weeks of pregnancy.

A $p$ value of $<0.05$ (two-tailed) was considered significant. Given the large number of statistical tests performed, no correction for multiplicity was applied in order to maintain sufficient power. Analyses were performed by A. Laenen using SAS software (Cary, NC, USA, version 9.4).

\section{Results}

Stratification of GDM subtypes The cohort consisted of 1813 women with both GCT and OGTT results available. Women without GDM were stratified into an abnormal GCT NGT group with 472 women (26.1\% of the total cohort) and a normal GCT NGT group with 1113 women $(61.9 \%$ of the total cohort). Based on a Matsuda index below P50 of normal GCT NGT women, women with GDM were classified into a GDM insulin-resistant group (189 [82.9\%] women) and a GDM insulin-sensitive group (39 [17.1\%] women). Additional stratification of GDM was performed into four subgroups according to Matsuda indices of $<$ P25, P25-P50, P50-P75 and >P75 compared with women with NGT (ESM Tables 1-3), identifying, respectively, a GDM high insulinresistant group (150 [65.8\%] of the women with GDM), GDM moderate insulin-resistant group (39 [17.1\%]), GDM moderate insulin-sensitive group (29 [12.7\%]) and GDM high insulin-sensitive group (10 [4.4\%]).

Characteristics Compared with women with NGT, women in the GDM insulin-resistant group were older, more often from an ethnic minority background, more likely to smoke before and during pregnancy, and more often had a first-degree family history of diabetes, a history of GDM and a history of impaired glucose intolerance (Table 1). They also had lower levels of education and a higher BMI, waist circumference, BP, FPG, fasting baseline total cholesterol, LDL-cholesterol and triacylglycerols, and a significantly higher increase in triacylglycerols between early pregnancy and at the time of the OGTT (Table 1). Compared with women with NGT, women in the GDM insulin-sensitive group more often had a firstdegree family history of GDM and a history of GDM, but had a significant lower BMI and similar BP, FPG and fasting lipid levels in early pregnancy (Table 1). When evaluating four GDM subgroups based on the Matsuda index, the GDM subgroup with the highest insulin resistance (Matsuda index $<$ P25 compared with women with NGT), had the most adverse metabolic risk profile compared with women with NGT (ESM Table 1).

Degree of hyperglycaemia FPG in early pregnancy was significantly higher in women with GDM with high insulin resistance compared with the GDM insulin-sensitive group 


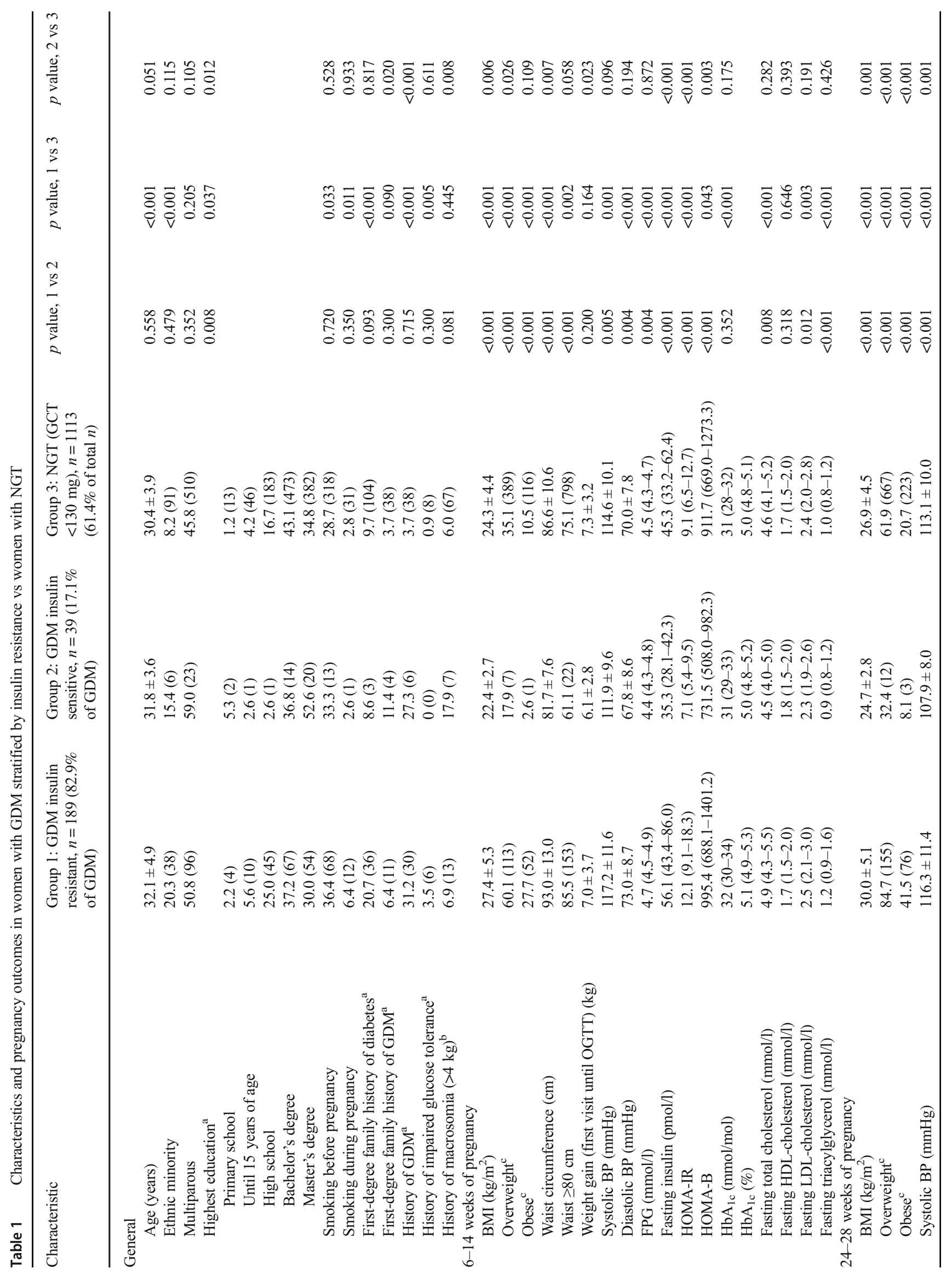




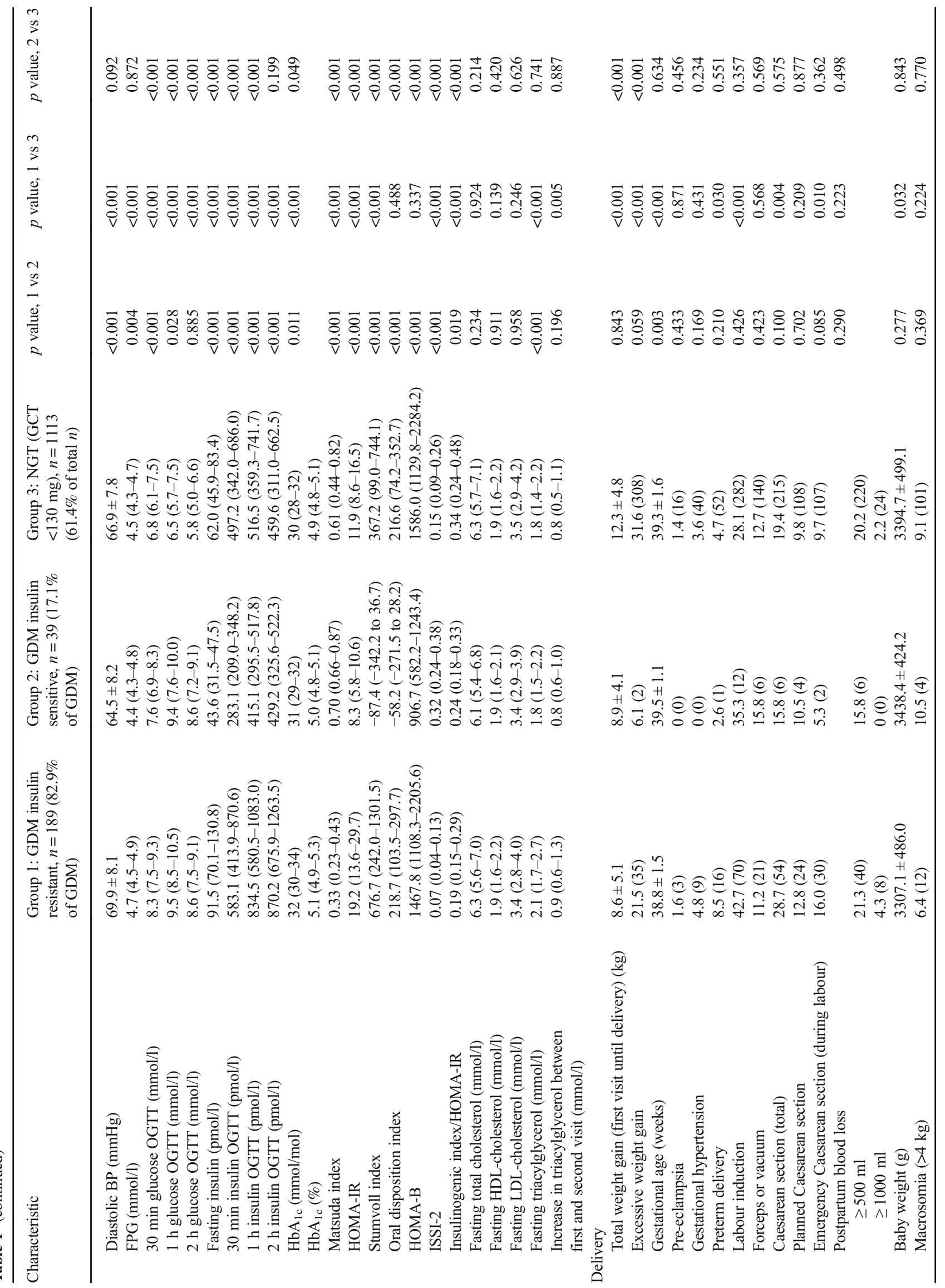




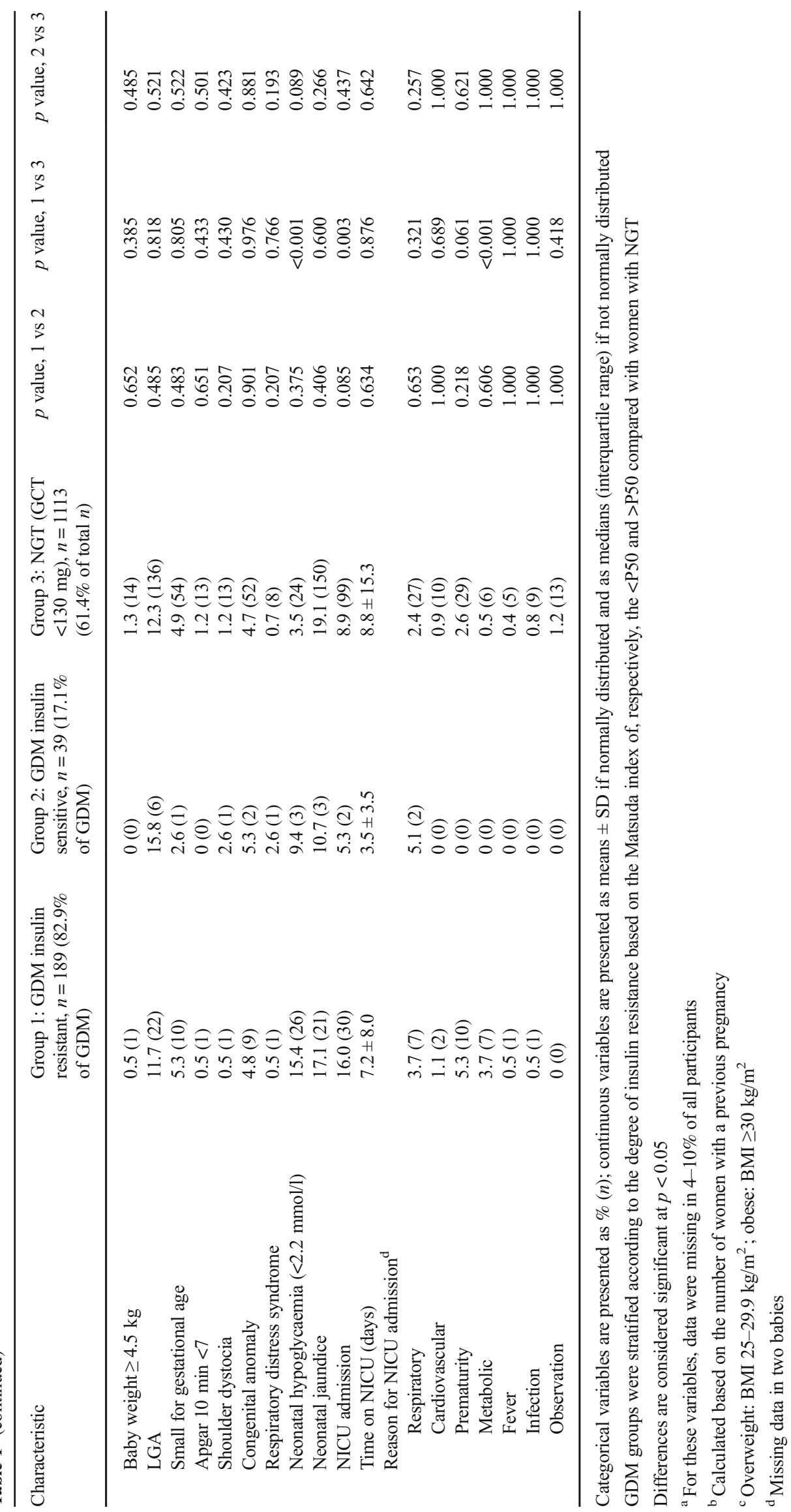


(Table 1). At the time of the OGTT, FPG, 30 min glucose levels and $1 \mathrm{~h}$ glucose levels were significantly higher in women with GDM with high insulin resistance compared with the GDM insulin-sensitive group, with similar $2 \mathrm{~h}$ glucose levels (Table 1). Both GDM groups had impaired beta cell function compared with women with NGT (Table 1). When evaluating four GDM subgroups based on the Matsuda index, the GDM subgroup with the highest insulin resistance (Matsuda index $<\mathrm{P} 25$ compared with women with NGT) had the highest FPG in early pregnancy and the highest FPG, $30 \mathrm{~min}$ and $1 \mathrm{~h}$ glucose levels at the time of the OGTT (ESM Table 1).

Of all women with GDM, 33 (14.5\%) needed treatment with insulin during pregnancy. The number of women receiving insulin, gestational age at the start of insulin treatment and the total insulin dose at the end of pregnancy did not differ significantly between the two GDM subtypes (Table 2). There were also no differences when women with GDM were stratified into four subgroups based on the Matsuda index (ESM Table 2).

Pregnancy outcomes Comparing the general GDM population with women with NGT showed that the frequencies of LGA infants, preterm deliveries, hypertensive disorders and NICU admissions were similar, but that rates of labour inductions, Caesarean sections and neonatal hypoglycaemia remained significantly higher among women with GDM compared with women with NGT (ESM Table 4).
Compared with women with NGT, women with GDM and insulin resistance had higher rates of preterm delivery $(8.5 \%$ vs $4.7 \%, p=0.030)$, labour induction $(42.7 \%$ vs $28.1 \%$, $p<0.001$ ), Caesarean section (total Caesarean sections: $28.7 \%$ vs $19.4 \%, p=0.004$; emergency Caesarean sections: $16.0 \%$ vs $9.7 \%, p=0.010)$, neonatal hypoglycaemia $(15.4 \%$ vs $3.5 \%, p<0.001)$ and NICU admissions $(16.0 \%$ vs $8.9 \%$, $p=0.003$ ) (Table 1). In the multivariable logistic regression analyses, using different models to adjust for demographics, BMI, FPG, $\mathrm{HbA}_{1 \mathrm{c}}$, lipid levels and gestational weight gain in early pregnancy, preterm delivery (OR 2.41 [95\% CI 1.08, 5.38]) and neonatal hypoglycaemia (OR 4.86 [95\% CI 2.04, 11.53]), remained significantly higher in women with GDM and insulin resistance compared with women with NGT (Table 3). Insulin-sensitive women with GDM had similar pregnancy outcomes as women with NGT (Table 1). When evaluating four GDM subgroups based on the Matsuda index, the GDM subgroup with the highest insulin resistance (Matsuda index <P25 compared with women with NGT), had the highest risk of several adverse pregnancy outcomes (inductions, preterm delivery, Caesarean section, neonatal hypoglycaemia and NICU admissions) compared with women with NGT (ESM Table 1). In multivariable logistic regression analyses, the Caesarean section rate (total and emergency Caesarean section), preterm delivery and neonatal hypoglycaemia remained significantly higher in this group compared with women with NGT (ESM Table 3). The GDM moderate insulin-resistant group (Matsuda index with P25-50 compared with women with NGT) more often had
Table 2 Insulin need during pregnancy and risk of glucose intolerance in the early postpartum period in women with GDM stratified by insulin resistance

\begin{tabular}{llll}
\hline Variable & $\begin{array}{l}\text { Group 1: GDM insulin } \\
\text { resistant, } n=189 \\
(82.9 \% \text { of GDM })\end{array}$ & $\begin{array}{l}\text { Group 2: GDM insulin } \\
\text { sensitive, } n=39 \\
(17.1 \% \text { of GDM })\end{array}$ & $p$ value \\
\hline $\begin{array}{l}\text { Treatment with insulin } \\
\text { Total on insulin }\end{array}$ & $14.8(28)$ & $12.8(5)$ & 0.649 \\
$\quad$ Basal bolus & $5.3(10)$ & $5.1(2)$ & \\
$\quad$ Only short-acting insulin & $5.8(11)$ & $7.7(3)$ & 0.858 \\
$\quad$ Only long-acting insulin & $3.7(7)$ & $0(0)$ & 0.379 \\
Gestational age at start of insulin (weeks) & $29.8 \pm 2.4$ & $29.8 \pm 1.9$ & 0.435 \\
Insulin dose at end of pregnancy (U) & $28.0 \pm 10.3$ & $25.8 \pm 21.2$ & 0.554 \\
6-16 weeks postpartum & & & 0.527 \\
Received postpartum OGTT & $82.0(155)$ & $87.2(34)$ & 0.722 \\
Time of OGTT (weeks) & $14.4 \pm 4.2$ & $14.8 \pm 3.7$ & \\
Glucose intolerance & $19.3(30)$ & $14.7(5)$ & \\
IFG & $7.1(11)$ & $5.9(2)$ & \\
IGT & $11.0(17)$ & $2.9(2)$ & \\
IFG + IGT & $1.3(2)$ & &
\end{tabular}

Categorical variables are presented as $\%(n)$; continuous variables are presented as means $\pm \mathrm{SD}$

The $\chi^{2}$ test was used for comparing groups on categorical variables, and the Kruskal-Wallis test was used for comparing multiple groups on continuous variables

IFG, impaired fasting glucose; IGT, impaired glucose tolerance 
Table 3 Risk of adverse pregnancy outcomes in the GDM insulin-resistant group vs women with NGT

\begin{tabular}{|c|c|c|c|c|c|c|}
\hline Variable & Model 1 & Model 2 & Model 3 & Model 4 & Model 5 & Model 6 \\
\hline Labour induction & $\begin{array}{c}1.74(1.26,2.41), \\
p<0.001\end{array}$ & $\begin{array}{c}1.71(1.19,2.45), \\
p=0.004\end{array}$ & $\begin{array}{c}1.61(0.99,2.62), \\
p=0.056\end{array}$ & $\begin{array}{c}1.37(0.81,2.32) \\
p=2.334\end{array}$ & $\begin{array}{c}1.56(0.90,2.70) \\
p=0.114\end{array}$ & $\begin{array}{c}1.51(0.98,2.32) \\
p=0.107\end{array}$ \\
\hline $\begin{array}{l}\text { Caesarean section } \\
\text { (overall) }\end{array}$ & $\begin{array}{c}1.67(1.18,2.37) \\
p=0.004\end{array}$ & $\begin{array}{c}1.54(1.04,2.29) \\
\quad p=0.031\end{array}$ & $\begin{array}{c}1.74(0.10,3.05), \\
\quad p=0.052\end{array}$ & $\begin{array}{c}1.68(0.91,3.10) \\
p=0.094\end{array}$ & $\begin{array}{c}1.43(0.75,2.71) \\
p=0.277\end{array}$ & $\begin{array}{c}1.36(0.86,2.17) \\
p=0.190\end{array}$ \\
\hline $\begin{array}{l}\text { Emergency } \\
\text { Caesarean section }\end{array}$ & $\begin{array}{c}1.77(1.14,2.75), \\
p=0.010\end{array}$ & $\begin{array}{c}1.72(1.03,2.86), \\
p=0.037\end{array}$ & $\begin{array}{c}1.99(0.88,4.50), \\
p=0.098\end{array}$ & $\begin{array}{l}1.85(0.75,4.55), \\
p=0.183\end{array}$ & $\begin{array}{c}1.50(0.57,3.96) \\
p=0.415\end{array}$ & $\begin{array}{c}1.57(0.86,2.90), \\
p=0.143\end{array}$ \\
\hline Preterm delivery & $\begin{array}{c}1.89(1.05,3.38), \\
p=0.033\end{array}$ & $\begin{array}{l}2.19(1.16,4.12), \\
\quad p=0.015\end{array}$ & $\begin{array}{c}3.04(3.00,3.08), \\
p<0.001\end{array}$ & $\begin{array}{c}6.66(2.10,21.16) \\
p=0.001\end{array}$ & $\begin{array}{c}3.44(3.39,3.49) \\
\quad p<0.001\end{array}$ & $\begin{array}{l}2.41(1.08,5.38), \\
\quad p=0.031\end{array}$ \\
\hline $\begin{array}{l}\text { Neonatal } \\
\text { hypoglycaemia }\end{array}$ & $\begin{array}{c}4.99(2.78,8.95), \\
p<0.001\end{array}$ & $\begin{array}{c}3.78(1.89,7.54), \\
p<0.001\end{array}$ & $\begin{array}{c}6.60(6.49,6.71), \\
p<0.001\end{array}$ & $\begin{array}{c}7.05(6.93,7.17) \\
p<0.001\end{array}$ & $\begin{array}{c}8.91(8.75,9.07) \\
\quad p<0.001\end{array}$ & $\begin{array}{c}4.86(2.04,11.53), \\
p<0.001\end{array}$ \\
\hline NICU admission & $\begin{array}{c}1.94(1.25,3.03), \\
p=0.003\end{array}$ & $\begin{array}{c}1.89(1.15,3.11), \\
p=0.012\end{array}$ & $\begin{array}{c}2.40(1.12,5.12), \\
\quad p=0.024\end{array}$ & $\begin{array}{l}2.68(1.18,6.07), \\
\quad p=0.019\end{array}$ & $\begin{array}{l}2.41(1.03,5.64), \\
\quad p=0.043\end{array}$ & $\begin{array}{c}1.64(0.88,3.06) \\
p=0.116\end{array}$ \\
\hline
\end{tabular}

Values are OR $(95 \% \mathrm{CI}), p$ value

Model 1: unadjusted

Model 2: adjusted for demographics (centre, age, ethnicity, parity, education and smoking before pregnancy)

Model 3: model $2+$ adjusted for first-degree family history of diabetes, previous history of GDM and BP in early pregnancy

Model 4: model 3 + adjusted for maternal BMI and waist circumference in early pregnancy

Model 5: model 4 + adjusted for fasting lipids (total cholesterol, LDL-cholesterol and triacylglycerols), FPG and $\mathrm{HbA}_{1 \mathrm{c}}$ in early pregnancy

Model 6: model $5+$ adjusted for gestational weight gain until the OGTT at 26-28 weeks of pregnancy

neonatal hypoglycaemia and NICU admissions compared with women with NGT, while the other GDM subgroups with less insulin resistance had similar pregnancy outcomes as women with NGT (ESM Table 1).

Glucose intolerance postpartum Of all women with GDM, $82.9 \%$ (189) received a postpartum OGTT at a mean \pm SD of $14 \pm 4$ weeks, and this was similar across the two GDM subtypes. Of all women with GDM who received a $75 \mathrm{~g}$ OGTT postpartum, none had diabetes and 35 (18.5\%) had glucose intolerance (impaired fasting glucose, impaired glucose tolerance or both), and this was not significantly different across the two GDM subtypes (Table 2). It also did not differ when the women with GDM were stratified into four subgroups based on the Matsuda index (ESM Table 1).

\section{Discussion}

We have shown that GDM subtypes with different degrees of insulin resistance have distinct phenotypes and differential risks of adverse pregnancy outcomes. The majority of women with GDM in our cohort had high insulin resistance. We demonstrate that GDM with a high insulin resistance represents a more adverse metabolic profile, with higher degrees of hyperglycaemia, BMI, BP and lipid levels, and a greater risk of adverse pregnancy outcomes than seen in women with NGT. In addition, women with GDM with high insulin resistance had also a higher degree of hyperglycaemia both early and later in pregnancy compared with women with GDM with less insulin resistance.

Previous small studies have suggested that GDM is a heterogeneous disease, mostly related to differences in BMI [4, 5, 17]. The North American study of Powe et al in a predominantly white population suggested a greater risk of adverse pregnancy outcomes in women with GDM with impaired insulin sensitivity [5]. In that study, women with impaired insulin sensitivity had a significantly higher rate of Caesarean sections and larger infants compared with women with NGT, which persisted after adjustment for BMI. This is in contrast to our results, since we found that women with high insulin resistance had similar rates of LGA infants and fetal macrosomia but significantly higher rates of preterm delivery, labour inductions, Caesarean sections, neonatal hypoglycaemia and NICU admissions compared with women with NGT. The total number of Caesarean sections in the high insulin resistance group vs the NGT group was relatively similar between the Powe et al study (33.3\% vs $15.2 \%)$ and our data $(28.7 \%$ vs $19.4 \%)$. In contrast, the rates of LGA infants $(11.7 \%)$ and neonatal hypoglycaemia $(15.4 \%)$ in the high insulin resistance group were lower in our study than in the Powe et al study (26.5\% and $23.5 \%$, respectively) [5]. In addition, after adjustment for several variables such as BMI, FPG and lipid levels in early pregnancy, the risks of preterm delivery and neonatal hypoglycaemia remained significantly higher in the high insulin resistance group compared with the NGT group in our study. Our data suggest, therefore, that the increased risk of adverse outcomes in women with GDM with high insulin resistance cannot be entirely explained by a 
higher BMI, FPG and fasting lipid levels in early pregnancy. At this point, we can only speculate on possible additional mechanisms of the increased risk of adverse complications in women with GDM with impaired insulin sensitivity. Previous studies have identified biomarkers involved in specific metabolite groups and several pathways in women with GDM, including amino acid metabolism, steroid hormone biosynthesis, glycerophospholipid metabolism and fatty acid metabolism. Further research is warranted to determine if metabolomics will result in new indicators for the management of GDM and GDM-related complications [18]. Differences in outcomes between GDM subtypes might also be related to differences in (epi)genetic susceptibility. Differences between our study and that of Powe et al may be related to population differences, since our sample size was much larger and had a higher prevalence of GDM and of women from an ethnic minority background. In addition, we had more clinical and biochemical variables available in early and later pregnancy for adjustment of outcomes.

We have also shown that women with high insulin resistance had higher baseline triacylglycerol levels and a significantly higher increase in triacylglycerols between early pregnancy and the time of the OGTT. There is increasing evidence that besides maternal BMI, gestational weight gain and hyperglycaemia, increased triacylglycerol levels (fasting and postprandial) early in pregnancy independently increase the risk of newborn adiposity $[19,20]$. Our study showed no differences in the rate of LGA infants between women with GDM and those with NGT. However, we did not have data on newborn adiposity.

The study of Powe et al stratified different physiological GDM subtypes according to insulin sensitivity and secretion below P25 in women with NGT by using the Matsuda and Stumvoll indices [5]. The Matsuda and Stumvoll indices have been used to calculate the glucose disposition index [21]. However, this has been criticised since a true rectangular hyperbolic relationship between insulin sensitivity and insulin secretion has only been established for the ISSI-2 and the insulinogenic index/fasting insulin [22, 23]. Moreover, all women with GDM (across all degrees of insulin resistance) have impaired beta cell dysfunction compared with women with NGT, as also shown by the different measures of insulin secretion in our study. Since there is a continuous relationship between the degree of hyperglycaemia and adverse pregnancy outcomes, the definition of GDM is based on consensus and any stratification of GDM into different subtypes will inherently be limited due to the classification of a continuous variable into categories. However, we feel that stratification of GDM based on underlying physiological processes adds to our knowledge on differences in phenotype and pregnancy complications across different GDM subtypes based on different degrees of insulin resistance. This might help to better tailor the management of GDM during pregnancy and at long-term follow-up after delivery to prevent complications.
Despite differences in phenotype and underlying insulin sensitivity, the rate of glucose intolerance in the early postpartum period was not significantly different across the different GDM subtypes in our study. This might be related to the overall small population of women with GDM in our study. The overall rate of glucose intolerance in the early postpartum period was $18.5 \%$, which is much lower than the rate of $42.2 \%$ that we previously reported in women with GDM diagnosed using a two-step screening strategy with a GCT and the 2013 WHO criteria [24]. The use of a universal one-step diagnostic approach with the $75 \mathrm{~g}$ OGTT and the 2013 WHO criteria for GDM probably results in a greater proportion of women being diagnosed with mild forms of GDM, and these women might have less impaired beta cell dysfunction than women who are diagnosed using both a GCT and OGTT. Long-term data are needed to evaluate whether the long-term risk of developing type 2 diabetes and an adverse cardiometabolic profile differs across GDM subtypes, as this might help to develop more targeted prevention programmes.

Here, we have presented the largest study to date on the characteristics of and pregnancy outcomes across different physiological GDM subtypes based on different degrees of insulin resistance. Moreover, women without GDM could be further stratified into those with an abnormal GCT and those with a normal GCT. In addition, we provide data on the risk of glucose intolerance in the early postpartum period. Limitations of the study are the small sample sizes for the GDM subgroups and the lack of a control group to compare postpartum OGTT results. In addition, we have no data on the long-term postpartum risk of glucose intolerance.

In conclusion, we have shown that different GDM subtypes have distinct phenotypes and differential risks of adverse pregnancy outcomes. GDM with high insulin resistance represents a more adverse metabolic profile with a greater risk of adverse pregnancy outcomes than those seen in women with NGT. In addition, insulin-sensitive women with GDM had a similar phenotype and similar pregnancy outcomes as women with NGT. There were no differences in the need for insulin treatment during pregnancy or in the rate of glucose intolerance in the early postpartum period between the different GDM subtypes.

Acknowledgements $\mathrm{KB}$ and $\mathrm{RD}$ are the recipients of a 'Fundamenteel Klinisch Navorserschap FWO Vlaanderen'. We thank I. Beckstedde (Department of Obstetrics, University Hospital of Antwerp, Belgium) and S. Van Imschoot (Department of Endocrinology, AZ St Jan Brugge, Belgium) for their help with participant recruitment and study assessments. We thank the research assistants, paramedics and physicians at all participating centres for their support, and we thank all of the women who participated in the study.

Data availability The datasets generated during and/or analysed during the current study are available from the corresponding author on reasonable request. 
Funding This investigator-initiated study was funded by the Belgian National Lottery, the Fund of Academic studies of UZ Leuven and the Fund Yvonne and Jacques François - de Meurs of the King Boudewijn Foundation. The sponsors of the study had no role in the design of the study or in the collection, handling, analysis or interpretation of the data, or in the decision to write and submit the manuscript for publication.

Duality of interest The authors declare that there is no duality of interest associated with this manuscript.

Contribution statement $\mathrm{KB}$, $\mathrm{PVC}$ and CMath conceived the project. CMoyso prepared the data and ALaen performed the statistical analysis. $\mathrm{KB}$ conducted the literature review. $\mathrm{KB}$ and CMath wrote the first draft of the manuscript. All authors contributed to the study design, including data collection, data interpretation and manuscript revision, and all authors have approved the final version. KB had full access to all of the data in the study and has final responsibility for the contents of the article and the decision to submit for publication.

\section{References}

1. Crowther CA, Hiller JE, Moss JR et al (2005) Effect of treatment of gestational diabetes mellitus on pregnancy outcomes. $\mathrm{N}$ Engl $\mathrm{J}$ Med 352(24):2477-2486. https://doi.org/10.1056/NEJMoa042973

2. Landon MB, Spong CY, Thom E et al (2009) A multicenter, randomized trial of treatment for mild gestational diabetes. N Engl J Med 361(14):1339-1348. https://doi.org/10.1056/NEJMoa0902430

3. Bellamy L, Casas JP, Hingorani AD, Williams D (2009) Type 2 diabetes mellitus after gestational diabetes: a systematic review and meta-analysis. Lancet 373(9677):1773-1779. https://doi.org/10. 1016/S0140-6736(09)60731-5

4. Catalano PM (2014) Trying to understand gestational diabetes. Diabet Med 31(3):273-281. https://doi.org/10.1111/dme.12381

5. Powe CE, Allard C, Battista MC et al (2016) Heterogeneous contribution of insulin sensitivity and secretion defects to gestational diabetes mellitus. Diabetes Care 39(6):1052-1055. https://doi.org/ $10.2337 / \mathrm{dc} 15-2672$

6. Benhalima K, Van Crombrugge P, Verhaeghe $J$ et al (2014) The Belgian Diabetes in Pregnancy Study (BEDIP-N), a multi-centric prospective cohort study on screening for diabetes in pregnancy and gestational diabetes: methodology and design. BMC Pregnancy Childbirth 14(1):226. https://doi.org/10.1186/1471-2393-14-226

7. Benhalima K, Van Crombrugge P, Moyson C et al (2018) The sensitivity and specificity of the glucose challenge test in a universal two-step screening strategy for gestational diabetes mellitus using the 2013 World Health Organization criteria. Diabetes Care 41(7):e111-e112. https://doi.org/10.2337/dc18-0556

8. Benhalima K, Van Crombrugge P, Moyson C et al (2018) A modified two-step screening strategy for gestational diabetes mellitus based on the 2013 WHO criteria by combining the glucose challenge test and clinical risk factors. J Clin Med 7(10):351. https://doi. org $/ 10.3390 / \mathrm{jcm} 7100351$

9. American Diabetes Association (2013) Standards of medical care in diabetes - 2013. Diabetes Care 36(Suppl 1):S11-S66. https://doi. org/10.2337/dc13-S011

10. Matthews DR, Hosker JP, Rudenski AS, Naylor BA, Treacher DF, Turner RC (1985) Homeostasis model assessment: insulin resistance and beta-cell function from fasting plasma glucose and insulin concentrations in man. Diabetologia 28(7):412-419. https://doi.org/ 10.1007/BF00280883

11. Matsuda M, DeFronzo RA (1999) Insulin sensitivity indices obtained from oral glucose tolerance testing: comparison with the euglycemic insulin clamp. Diabetes Care 22(9):1462-1470. https://doi.org/10.2337/diacare.22.9.1462

12. Kahn SE, Prigeon RL, McCulloch DK et al (1993) Quantification of the relationship between insulin sensitivity and beta-cell function in human subjects. Evidence for a hyperbolic function. Diabetes 42(11):1663-1672. https://doi.org/10.2337/diab.42.11.1663

13. Retnakaran R, Qi Y, Goran MI, Hamilton JK (2009) Evaluation of proposed oral disposition index measures in relation to the actual disposition index. Diabet Med 26(12):1198-1203. https://doi.org/ 10.1111/j.1464-5491.2009.02841.x

14. Stumvoll M, Van Haeften T, Fritsche A, Gerich J (2001) Oral glucose tolerance test indexes for insulin sensitivity and secretion based on various availabilities of sampling times. Diabetes Care 24(4):796-797. https://doi.org/10.2337/diacare.24.4.796

15. Devlieger HMG, Bekaert A, Eeckels R (2000) Standaarden van geboortegewicht-voor-zwangerschapsduur voor de Vlaamse boreling. Tijdschr voor Geneeskunde 56(1):1-14 [article in Dutch]. https://doi.org/10.2143/TVG.56.1.5000625

16. Rasmussen KM, Yaktine AL (eds) (2009) Weight gain during pregnancy: reexamining the guidelines. National Academies Press, Washington, DC

17. Cheney C, Shragg P, Hollingsworth D (1985) Demonstration of heterogeneity in gestational diabetes by a $400-\mathrm{kcal}$ breakfast meal tolerance test. Obstet Gynecol 65(1):17-23

18. Chen Q, Francis E, Hu G, Chen L (2018) Metabolomic profiling of women with gestational diabetes mellitus and their offspring: review of metabolomics studies. J Diabetes Complicat 32(5):512523. https://doi.org/10.1016/j.jdiacomp.2018.01.007

19. Barbour LA, Hernandez TL (2018) Maternal non-glycemic contributors to fetal growth in obesity and gestational diabetes: spotlight on lipids. Curr Diab Rep 18(6):37. https://doi.org/10.1007/s11892018-1008-2

20. Barbour LA, Farabi SS, Friedman JE et al (2018) Postprandial triglycerides predict newborn fat more strongly than glucose in women with obesity in early pregnancy. Obesity 26(8):13471356. https://doi.org/10.1002/oby.22246

21. Elbein SC, Wegner K, Kahn SE (2000) Reduced beta-cell compensation to the insulin resistance associated with obesity in members of Caucasian familial type 2 diabetic kindreds. Diabetes Care 23(2): 221-227. https://doi.org/10.2337/diacare.23.2.221

22. Retnakaran R, Hanley AJ, Raif N et al (2005) Adiponectin and beta cell dysfunction in gestational diabetes: pathophysiological implications. Diabetologia 48(5):993-1001. https://doi.org/10.1007/ s00125-005-1710-x

23. Retnakaran R, Shen S, Hanley AJ, Vuksan V, Hamilton JK, Zinman B (2008) Hyperbolic relationship between insulin secretion and sensitivity on oral glucose tolerance test. Obesity 16(8):19011907. https://doi.org/10.1038/oby.2008.307

24. Benhalima K, Jegers K, Devlieger R, Verhaeghe J, Mathieu C (2016) Glucose intolerance after a recent history of gestational diabetes based on the 2013 WHO Criteria. PLoS One 11(6): e0157272. https://doi.org/10.1371/journal.pone.0157272

Publisher's note Springer Nature remains neutral with regard to jurisdictional claims in published maps and institutional affiliations. 